\title{
Splenectomy at Caesarean: Management of Intractable ITP during Pregnancy
}

\author{
Paban Sharma', Paras Acharya ${ }^{2}$, Jaya Narayan Shah ${ }^{3}$, Rosani Amatya' \\ Dept Gyn/Obs ', Dept of Medicine², Dept. of Surgery ${ }^{3}$ \\ Patan Hospital
}

\begin{abstract}
Pregnancy with ITP is not a rare problem but sometime it is difficult to manage the condition because the decreased platelet count can lead to significant morbidity and even mortality due to bleeding during delivery and in post natal period. It is also related to neonatal thrombocytopenia which again can be dangerous at times. Here, we present a similar case of severe thrombocytopenia during pregnancy (ITP) and managed successfully by doing Splenectomy during caesarean section.
\end{abstract}

Key words: Immune thrombocytopenic purpura (ITP), pregnancy, splenectomy

\section{Introduction}

Immune thrombocytopenia is a condition where $\operatorname{IgG}$ antibodies are produced against the platelet membrane glycoproteins, which leads to accelerated destruction of antibody-coated platelets in the reticuloendothelial system (RES), mainly in the spleen. This disease occurs more in female and during fertile period. Due to decreased platelet, patient will have bleeding tendency, which is detrimental in relation to menstrual blood flow and delivery process. If not managed properly, there is high chance of bleeding during the labor process. Steroid is the mainstay of treatment followed by immunoglobulin and sometime even splenectomy is needed to save the life.

\section{Case}

A 28 yrs lady recently married, from eastern Nepal but residing in Kathmandu presented (2064.2.16) in a private clinic of the Patan Hospital with the history of P/V bleeding for last 10 days. She had a feeling of weakness and palpitation. On physical examination, she was pale but other findings were within normal limits. On per speculum examination there was significant $\mathrm{P} / \mathrm{V}$ bleeding, but size of the uterus was normal without adnexal mass. She had menarche at 13 years, regular period but heavy flow lasting for 7 days. She was diagnosed as a case of Immune thrombocytopenic purpura (ITP) 9 yrs back (2057BS) in BPKIHS Dharan and she needed transfused for anemia. Norethisterone was started and medical consultation was done and with the impression of chronic ITP with iron deficiency anemia due to chronic blood loss and treated with hematinic and steroid. She conceived without plan after 10 months. Prednisolone was restarted at 15 weeks of gestation due to decreasing platelet count. At 22 weeks of gestation, she needed platelet transfusion and further transfusion was done at 24 weeks of gestation. Her antenatal check up was normal and platelet count was above 50,000 till 33 weeks of gestation. At 33 weeks, again her platelet count dropped to critical level needing further transfusion of Platelet rich plasma (PRP). She was admitted at 35 weeks of gestation and further transfusion of PRP done. Her antenatal investigations were with in all normal limits including obstetric scan except she had high Glucose challenge test (GCT) but $100 \mathrm{gm}$ OGTT was normal. After a discussion with patient party, elective caesarean section along with Splenectomy was decided. Since she started leaking at 36 weeks along with unfavorable cervix, an elective caesarean delivery and splenectomy was done at $36^{+3 \text { days }}$ of gestation after making platelet count above 75,000/ cumm with 18 units of PRP in stock. Both intra and post operative periods were uneventful and operative

Correspondence

Dr. Paban sharma, $M D$,

Consultant, Gyn/Obs department, Patan Hospital

Mobile : 9851097844

E-mail:paban.Sharma@gmail.com 
blood loss was not that significant. Patient was kept in ICU for close observation and her platelet count went up to 95,000 in immediate post-operative period and became normal next day. She was vaccinated against pneumococcus and meningococcus. She was discharged on 9th post operative day. Platelet count in the new born was within normal limits.

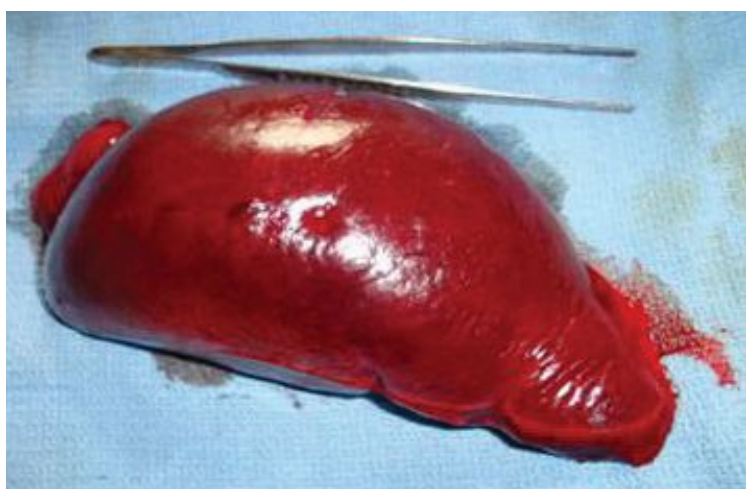

Fig 1. Splenectomy specimen

\section{Comment}

Normal platelet count in human being is 150 $400,000 /$ cum. If it is less than 50,000 it is severe thrombocytopenia. The mean platelet count in pregnant women is slightly less than that of non pregnant women. Thrombocytopenia is related to either decreased production or increased destruction. Increased platelet destruction can be caused by an immunologic destruction, abnormal platelet activation, or platelet consumption resulting from excessive bleeding or exposure to abnormal vessels. Problem of less production is less common and is usually associated with either folate deficiency, leukemia or aplastic anemia ${ }^{1}$.

Thrombocytopenia usually complicates around $10 \%$ of all pregnancies. Two third of such cases are due to gestational thrombocytopenia (essential thrombocytopenia), followed by pre-eclampsia (21\%) and the Immune thrombocytopenia (ITP) is related to around $4 \%$ of cases $^{2}$. Gestational thrombocytopena is asymptomatic, platelet never fall below the critical level and it disappears in post partum period.

Other causes of thrombocytopenia in pregnancy includes Systemic lupus Erythematosus, Antiphospholipid syndrome, Thrombotic thrombocytopenic purpura, Hemolytic uremic syndrome, Human immunodeficiency virus (HIV) infection, Disseminated intravascular coagulation, Hypersplenism, Congenital thrombocytopenias, Pseudothrombocytopenia (laboratory artifact) and sometime it is related to various medicine intake like heparin, quinine, quinidine, zidovudine, sulfonamides etc.
Immune thrombocytopenic purpura (ITP) is a disorder where the patient produces IgG antiplatelet antibodies that recognize platelet membrane glycoproteins, which leads to accelerated destruction of antibodycoated platelets in the reticuloendothelial system (RES), mainly in the spleen. There are no definite pathognomonic signs, symptoms, or diagnostic tests for ITP; it is a diagnosis of exclusion. However, four findings have been traditionally associated with the condition: 1) persistent thrombocytopenia (platelet count $<100,000 / \mathrm{mL}$ with or without accompanying megathrombocytes on the peripheral smear), 2) normal or increased numbers of megakaryocytes determined from bone marrow, 3) exclusion of other systemic disorders or drugs that are known to be associated with thrombocytopenia, and 4) absence of splenomegaly ${ }^{1}$. Most of the women with ITP will give a history of bruising easily, petechiae, epistaxis and gingival bleeding, but many are asymetomatic. Hemorrhagic problem rarely occur unless the platelet count is less than $20,000 / \mathrm{mL}$. Generally, It is believed that the course of ITP usually is not affected by pregnancy, although there have been anecdotal reports of patients' conditions worsening during pregnancy and improving postpartum. Pregnancy may be adversely affected by severe thrombocytopenia, mainly during intrapartum and immediate post-partum period causing heavy bleeding and sometime fetus may be affected. Maternal IgG antiplatelet antibodies can cross the placenta, placing the fetus and neonate at risk for the development of thrombocytopenia. Retrospective case series of ITP in pregnancy indicate that $12-15 \%$ of infants born to mothers with ITP will develop platelet counts less than $50,000 / \mathrm{mL}$. However, it is difficult to predict which neonates are at risk of severe thrombocytopenia ${ }^{3}$ ITP is also significantly and independently associated with preterm delivery before 34 weeks gestation and with perinatal mortality ${ }^{4}$. Similarly, higher rates of placental abruption were found in pregnant women with thrombocytopenia ${ }^{5}$.

Chronic ITP typically occurs in the second or third decade of life and has a female to male ratio of 3:1. Estimates of the frequency of ITP during pregnancy vary widely, affecting one in 1,000-10,000 pregnancies $^{1}$.

The goal of treatment during pregnancy is to raise the platelet count into a hemostatically safe range. The need for treatment will depend on the platelet count and whether there is bleeding. Apart from platelet transfusion, Corticosteroids are the first line of therapy in pregnant women; intravenous immune globulin is commonly used in steroid resistant patients. The drugs like dapsone, and danazol have been tried successfully to manage the condition ${ }^{6}$. Other treatment includes the use of intravenous anti D immunoglobulin, 
Splenectomy during pregnancy and intrapartum period $^{6}$. Splenectomy is considered acceptable for patients with refractory immune thrombocytopenic purpura and severe thrombocytopenia. Conventionally laparoscopic procedure during pregnancy is not done usually but Laparoscopic Splenectomy has been done safely during pregnancy in such cases ${ }^{7,8}$. Since, the labor process is unpredictable, planned delivery is needed to prevent disastrous complication of bleeding during intrapartum period, it may be necessary to do elective caesarean delivery. This may be related to prevent fetal intracranial hemorrhage?

In our case, patient was on steroid from the very beginning. She received several units of platelet transfusion to maintain platelet level. During third trimester, platelet count went below the critical level despite of medical management. So, Splenectomy was decided during a planned caesarean section. Platelet was transfused to increase the platelet level above 50,000 during operation. After splenectomy platelet count increased without any platelet transfusion and there was no excessive bleeding during or after surgery. Now the patient is having normal platelet count without any medicine.

\section{Conclusion}

Severe ITP during pregnancy may across in our day to day practice and if not managed adequately can have significant morbidity and even mortality to the patient. Splenectomy is one of the and perhaps a last modality of treatment to manage such case until now.

\section{References}

1. ACOG practice bulletin

2. Federici L, Serraj K, Maloisel F, Andrès E. Thrombocytopenia during pregnancy: from etiologic diagnosis to therapeutic management.
Presse Med 2008 Sep; 37(9):1299-307. Epub 2008 Jul 14.

3. Howman RA, Barr AL, Shand AW, Dickinson JE. Antenatal intravenous immunoglobulin in chronic immune thrombocytopenic purpura: case report and literature review. Fetal Diagn Ther 2009; 25(1):93-7. Epub 2009 Feb 14.

4. Belkin A, Levy A, Sheiner E. Perinatal outcomes and complications of pregnancy in women with immune thrombocytopenic purpura. J Matern Fetal Neonatal Med 2009 Nov; 22(11):1081-5.

5. Parnas M, Sheiner E, Shoham-Vardi I, Burstein E, Yermiahu T, Levi I, Holcberg G, Yerushalmi R. Moderate to severe thrombocytopenia during pregnancy. Eur J Obstet Gynecol Reprod Biol. 2006 Sep-Oct; 128(1-2):163-8. Epub 2006 Mar 13.

6. Varghese L, Viswabandya A, Mathew AJ. Dapsone, danazol, and intrapartum splenectomy in refractory ITP complicating pregnancy. Indian J Med Sci 2008 Nov; 62(11):452-5.

7. Sukenik-Halevy R, Ellis MH, Fejgin MD.Management of immune thrombocytopenic purpura in pregnancy. Obstet Gynecol Surv 2008 Mar; 63(3):182-8.

8. Gernsheimer T, McCrae KR. Immune thrombocytopenic purpura in pregnancy. Curr Opin Hematol 2007 Sep; 14(5):574-80.

9. Elezović I, Bosković D, Tomin D, Pilić Z, Miković Z, Popović M, Knezević S, Sukalo M. Klinika za ginekologiju i akuserstvo, Beograd. Cesarean section combined with splenectomy in severely resistant immune thrombocytopenia. Acta Chir Iugosl 2002; 49(3):51-4.

10. Paniel BJ, Truc JB, Poitout P. Autoimmune thrombocytopenia and pregnancy. J Gynecol Obstet Biol Reprod (Paris) 1987; 16(1):17-26. 